\title{
La adquisición del rol y la identidad sexual: función de la familia
}

\section{Félix López *}

Universidad de Salamanca

1. REVISION DE LA

LITERA TURA, PLANTEAMIENTO DEL PROBLEMA Y OBJETIVOS DEL TRABAJO

Si bien hasta los años cincuenta sólo la corriente psicoanalítica se había ocupado de estos temas, durante las últimas décadas se han multiplicado los trabajos dedicados al estudio del desarrollo de la identidad de género y el rol sexual. Las corrientes psicoanalíticas, conductistas, e incluso algunos autores piagetianos, han hecho numerosas publicaciones.

Pero las diferentes metodologías usadas, la falta de coincidencias en terminología y, sobre todo, los distintos constructos teóricos desde los que se interpretan los datos, han hecho que, a pesar de la gran cantidad de datos recogidos, se mantengan las diferencias.

En efecto, los autores de unas y otras corrientes no coinciden en la descripción del proceso de adquisición de la identidad y el resol sexual, y aun menos en las explicaciones de dicho proceso (López $F$, 1980):

- Para el psiconálisis, llegada la fase genital, el niño estaría biológicamente orientado a preferir sexualmente al pro- genitor del sexo opuesto, mientras tiene sentimientos ambivalentes para con el progenitor del propio sexo. El imiedo a la castración y el miedo a perder el objeto amado le hace renunciar a sus sèntimientos de rivalidad con el progenitor del propio sexo y a llevar a cabo un prónceso de identificación con su figura (deseo de estar en su lugar, ser como él, etc.)! Identificación con el progenitor del propio sexo e interiorización de él, son los mecanismos centrales del proceso.

Esta explicación, como es sabido, presupone la universidad del complejo de Edipo y la existencia de un deseo especifico de naturaleza sexual para que dicho proceso tenga lugar. Ambos presupuestos están lejos de ser probados.

Por otra parte, si tomamos en serio la cronología de la escuela psicoanalítica ortodoxa, este proceso tendría lugar entre los tres y los seis años aproximadamente. Como veremos más adelante, ałlos 3 años los niños ya tienen una clara conciencia de su identidad y rol sexual.

- Para Kohlberg, autor de orientación piagetiana, la adquisición de la identidad y el rol sexual no están determinadas por la programación biológica ni por las influencias ambientales, sino por la organización

* Dirección del autor: Universidad de Salamanca. Facultad de Filosotia y CC. EE. Séçıōn đẹ Psıcólógia. Salamanca. 
cognitiva del propio niño. A medida que van cambiando las estructuras cognitivas lo hacen también las actitudes sexuales. $\mathrm{Y}$, precisamente por ello, a patrones universales de evolución cognitiva corresponden patrones universales de evolución en la adquisición de la identidad y el rol sexual.

El proceso podría resumirse así:

1. El niño hace un juicio simple y básico de su identidad sexual: «soy niño» o «soy una niña».

2. Organiza sus actitudes sexuales a partir de ese juicio: tiende a dar valor positivo a lo referido a su propio sexo.

3. Así se generaría también la identificación. Soy como mi "padre" o mi «madre».

Aunque este juicio cognitivo lo hace el niño en el segundo o tercer año de vida, la conservación de la identidad de género no tendría lugar hasta los 6 ó 7 años (cuando adquiere el resto de las conservaciones).

Por nuestra parte, creemos con Kohlberg que el juicio cognitivo es un factor decisivo en el proceso: tal juicio es tan importante que una vez que el niño llega a él, podrá sentirse más o menos satisfecho con la propia identidad sexual y el propio rol, pero no puede negarlos.

Pero este juicio afecta menos al rol y no garantiza la identificación con el progenitor del mismo sexo, a no ser que la reduzcamos a mero juicio cognitivo: «soy de tu mismo sexo". La identificación no es un mero juicio cognitivo, sino una asimilación que implica una relación positiva en la que interviene el "deseo»: «quiero ser como tú». En este sentido el juicio cognitivo ayuda a inclinarse por unas u otras figuras de identificación, pero no garantiza que ésta tenga lugar. Incluso pueden darse identificaciones contrarias a la identidad sexual y adopciones del rol inverso.

- Para las teorías del aprendizaje social el proceso de sexuación no depende de impulsos biológicos ni de estructuras cognitivas, sino que, como escribe Walter, "en teoría del aprendizaje social pueden definirse las conductas sexualmente tipificadas como aquellas que proporcionan típicamente distinta gratificación a uno u otro sexo o, en otras palabras, tienen consecuencias que varían según el sexo del sujeto... (así): primero aprende a dis- tinguir los patrones de conducta sexualmente tipificados; después a generalizar estas experiencias... a situaciones nuevas $y$, finalmente, a practicar dicha conducta» (Bandura y Walter, 1965).

Este proceso se lleva a cabo por el aprendizaje a través de los paradigmas de condicionamiento clásico, operante y aprendizaje vicario.

Esta orientación aporta una indudable luz sobre la importancia del ambiente. Efectivamente, depende en buena medida con qué se asocie el sexo, qué consecuencias tengan determinados comportamientos sexualmente tipificados y de qué modelos sexuales se disponga, cómo se asumirá la propia identidad y rol sexual.

Pero tampoco se explica desde esta perspectiva todo el proceso de la identidad y rol sexual. Efectivamente, ni está suficientemente probado que se recompense de forma muy diferente a los niños de las niñas por manifestar un comportamiento sexual apropiado antes de los cinco años (Bee, 1977), ni por supuesto, el juicio cognitivo sobre la identidad sexual cambia en razón de las recompensas. En todo caso el ambiente tendría poder sobre el rol sexual, pero no sobre la identidad.

Más bien creemos que las recompensas ayudan a establecer el juicio de identidad $y$, especialmente, a que la autoclasificación se asuma satisfactoriamente facilitando la identificación con las figuras del propio sexo o, si los refuerzos son negativos respecto a la propia identidad sexual, a que no tenga lugar la identificación.

Ninguna, pues, de estas tres teorías tomadas por si solas proporcionan una explicación fehaciente del proceso de adquisición de la identidad y el rol sexual, aunque en todas ellas se encuentren elementos positivos para construir una explicación más verídica y completa.

Las diferencias terminológicas y conceptuales han contribuido a hacer estas discusiones teóricas más confusas. Mientras los psicoanalistas hablan casi siempre de identificación, los autores de orientación cognitiva hablan de identidad y los conductistas, de tipificación sexual, preferentemente. 
Tales términos implican conceptos distintos:

La identidad hace referencia a la autoclasificación como niño o como niña, primordialmente, mientras la identificación implica sentimientos de suplencia, deseos de ser como otro, imitación global de las conductas de otro (Papalia, 1979), etc. La tipificación sexual o asunción del rol que la sociedad asigna a cada sexo es algo bien distinto de ambos conceptos anteriores: se trata de asimilar las características conductuales propias de cada sexo en una sociedad determinada.

No es, pues, extraño que los que hablan desde el punto de vista de la identi- dad den prioridad al juicio cognitivo, los que lo hacen desde los estudios de «tipificación de conductas sexuales" crean prioritaria la historia de refuerzos y, por último, aquellos que se ocupan de los conflictos de elección de figuras de identificación... hablen de deseos de suplencia e interiorización.

Desde perspectivas teóricas distintas, constructos teóricos distintos y metodologías de investigación muy distantes, se estudian temáticas muy próximas, pero no siempre coincidentes. Normal es que entre estas corrientes no se haya pasado de un diálogo de sordos. Como además es frecuente que unos conceptos hayan

Teorias explicativas: adquisición de la identidad y rol sexual Esquema de Koblberg (1966)

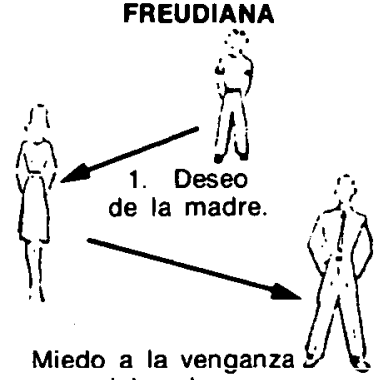
del padre.

2. Identificación con el padre

3. Identidad
APRENDIZAJE SOCIAL

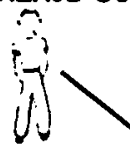

1. Adhesión al padre como máximo dispensador de recompensas (y castigos).

2. Identificación. Elección del padre como modelo.

3. Identidad sexualmente tipificada.
desaRROllo cogNItIVO

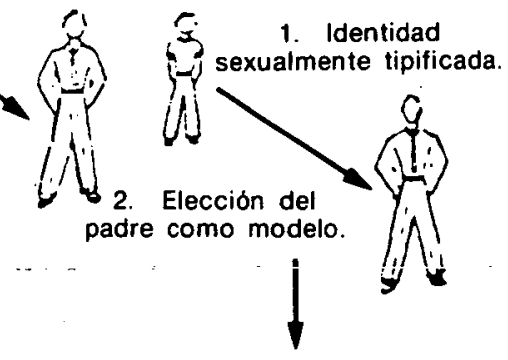

3. Adhesión

al padre.

\section{VALORACION}

Estas tres teorias parten de constructos distintos y estudian e interpretan los datos con metodologias diferentes. En realidad hablan de diferentes cosas y con lenguajes irreconciliables. 'La mayor deficiencia es la falta de distinción entre identidad, rol, identificación. Cada una de estas teorias habla fundamentalmente desde uno de estos conceptos generalizando los datos a todos ellos:

\section{Freud Identificación}

1. Pulsión

2. Conflicto edípico:

- deseo heterosexual

- miedo a castración

- identificación con el progenitor del mismo sexo
Kohlberg Identidad

- Actividad cognitiva

- Juicio intelectual sobre las diferencias

\section{Walter}

Rol

- Conducta en función de los estimulos

- Aprendizaje por:

- Asociación

- Instrumental

- Observación

Las 'tres teorias pueden ayudarnos a entender la influencia de tres factores distintos: factores bio-psicológicos del proceso de sexuación (psicoanálisis), factores cognitivos (piagetianos), factores sociales (aprendizaje social). Son en este sentido teorias complementarias que habria que unificar en una teoria global distinta que tuviera en cuenta todos estos ractores. Los datos aportados por Money sobre proceso de sexuacion apuntan en esta dirección, pero carece de una teoria psicológica. 
querido -reducirse a otros, el entendimiento se hace imposible y las discusiones teóricas inevitables:

En todo caso, interesa reseñar una coincidencia fundamental: la familia es el agente esencial en el proceso de adquisición. Si los modelos de identificación (en el psicoanálisis), los refuerzos y los modelos de imitación (en las teorías del aprendizaje social), o en menor grado, los modelos en los que reconoce su propia identidad (orientación cognitiva), no son adecuados, el proceso de adquisición puede estar amenazado y las consecuencias sobre la conducta sexual pueden ser graves.

Efectivamente, la familia no es solo el agente esencial, sino también el modelo de referencia para una buena aceptación de la identidad y rol que le ha tocado vivir.

En las últimas décadas una distinción fundamental ha clarificado este campo de discusión, Money (1975), Nedelman (1981):

- Identidad de género: autoclasificación como hombre o mujer, basada esencialmente en la figura corporal.

- Rol sexual: se refiere a los comportamientos, sentimientos, actitudes... que se consideran propios del hombre o de la mujer. Tiene, en este sentido, una base más cultural.

Entre ambos lo habitual es que exista una relación de adecuación armónica. Pero no siempre es así, como ocurre, por poner solo un ejemplo, en los casos de trasvestimos y transexualismo. $\mathrm{O}$ en el caso, menos extremo, del rechazo del papel que la sociedad asigna a la mujer. Algunos autores llegan a defender la idea de que los roles deberían ser abolidos (Bem, 1976; López, 1982).

A partir de esta distinción se han llevado a cabo numerosos trabajos sobre el proceso de adquisición de la identidad y el rol sexual. Como resultado de ellos podemos establecer con cierta claridad algunas conclusiones (Money, 1973; Thompson y Bentler, 1973; Thompson, 1975; Slaby y Frey, 1975; Emmerich, Goldman, Kirsh y Sharabany, 1977; Marcus y Overton, 1978; McCanaghy 1979; etc.):

- La adquisición de la identidad y rol sexual tiene lugar entre el año y medio y los tres años acabando hacia el tercero 0 cuarto año el período crítico de adquisición (Money, Hampson y Hampson, 1957; Money y Ehrhardt, 1972). El proceso de adquisición ha sido descrito con precisión por Thompson_(1975):

- primero aprende a reconocer que hay dos sexos: 0,18 ;

- en segundo lugar se incluye a sí mismo en una u otra categoría: $0,18-3,0$;

- usa el «rótulo» del sexo como guía de preferencias relacionadas con los roles sexuales: 3,0 .

- La permanencia de género no se adquiere -hasta los seis o siete años, cuando el niño es ya capaz de: conservar las cantidades líquidas, la sustancia, etc. (Kohlberg, 1966). Esta permanencia no tiene su origen en base a los genitales en un principio, como podía razonablemente esperarse, sino que está más en relación con las características del «rol». Se distingue, pues, de un período posterior en el que el género está determinado por los genitales en lugar de por las conductas dependientes del rol (llevar el pelo de una forma u otra, los vestidos, etcétera). A este período no llegaría el niño hasta los siete o nueve años (Thompson y Bentler, 1973; McGonaghy, 1979).

Pero son aún muchos los puntos oscuros, especialmente cuando se intentan interpretar estos datos: ¿de qué factores dependen estos procesos?; ¿qué relación existe entre la identidad de género y el rol?; ise puede hablar de que uno es anterior al otro?; ¿pueden comprobarse y generalizarse los estadios descritos por Thompson y Bentler (1973) y confirmados por McGonaghy (1979)?

\section{- Objetivos del trabajo}

Aun no pudiendo aclarar todos los problemas pendientes nos ha parecido útil emprender un trabajo referido a ellos con los siguientes objetivos:

1. Iniciar este campo de rinvestigación en España.

$2 .^{\circ}$ Intentar aportar alguna luz sobre:

- Los factores que determinan el desarrollo de la identidad de género y el rol sexual, toda vez que una cronología del desarrollo ya está fundamentalmente establecida -crono- 
logía que también: estudiamos.

- Comprobar los datos referidos a la permanencia de gé-" nero y a los dos estadios de. que. hablan los últimos- estudios, así como los factores que los determinan.

- Establecer las relaciones existentes entre rol-sexual e identidad de género.

\section{Metodología}

Seleccionamos una muestra estratificada por sexos, edades y circunstancias ambientales familiares: $\mathbf{N}=70: 38$ niñas $y$ 32 niños.

Las circunstancias ambientales usadas como criterio de selección fueron:

- Un grupo de niños (29) de familias de clase media-alta y con ambos padres trabajando fuera de casa.

- Un grupo de niños (25) de clase media-baja de familias en las que sólo trabaja el padre, mientras la madre permanecía en casa.

- Un grupo de niños (16) de clase baja y con una estructura familiar deficitaria: hijos de madres solteras, pa-
Los desequilibrios.en los sujetos de la muestra, que fue estructurada con todo cuidado; vienen dados porque en algunos casos no pudimos disponer de sujetos (por ejemplo, de niños y- niñas de más de seis años en el caso de las familias deficitarias) y en otros las condiciones naturales de los grupos. no nos permitieron hacer una estructuración estadísticamente perfecta. En todo caso, pensamos que la muestra está suficientemente equilibrada.

Esta muestra, por tanto; se compone de sujetos que difieren en edad, sexo y estructura familiar que eran, junto con otras variables que no hacen relación directa a nuestro tema, las que actuaban como «factores", cuya influencia en la adquisición de la identidad y el rol sexual se trataba de estudiar.

Este tipo de diseño puede ser catalogado de transversal por edades (Baltes y cols., 1981). Este diseño, a pesar de los límites que tiene, es uno de los más clásicos en psicología evolutiva. Nuevas aplicaciones longitudinales y transversales, combinadas, nos iran permitiendo superar dichos límites.

Para operacionalizar la identidad y rol sexuai, eiaboramos un instrumento de medida adaptado a los niños de esta edad:

\section{TABLA 1}

\section{Muestra}

\begin{tabular}{|c|c|c|c|c|c|c|c|}
\hline \multirow[b]{2}{*}{ Años } & \multicolumn{2}{|c|}{$\begin{array}{l}\text { (1) } \\
\text { Ambos padres } \\
\text { trabajando }\end{array}$} & \multicolumn{2}{|c|}{$\begin{array}{c}\text { (2) } \\
\text { Padre trabaja } \\
\text { madre en casa }\end{array}$} & \multicolumn{2}{|c|}{$\begin{array}{c}\text { (3) } \\
\text { Familia } \\
\text { «deficitaria» }\end{array}$} & \multirow[b]{2}{*}{ Totales } \\
\hline & $\begin{array}{c}\text { (a) } \\
\text { Varón }\end{array}$ & $\begin{array}{c}\text { (b) } \\
\text { Hembra }\end{array}$ & Varón & $\begin{array}{c}\text { (b) } \\
\text { Hembra }\end{array}$ & $\begin{array}{l}\text { (a) } \\
\text { Varón }\end{array}$ & $\begin{array}{c}\text { (b) } \\
\text { Hembra }\end{array}$ & \\
\hline 2 & 1 & 2 & 1 & 2 & 2 & 1 & 9 \\
\hline $2-3$ & 2 & 1 & 2 & 2 & $i$ & 2 & 10 \\
\hline $3-4$ & 2 & 2 & 2 & $\bar{l}$ & 2 & 1 & 10 \\
\hline $4-5$ & 2 & 3 & 2 & 2 & 2 & 2 & 13 \\
\hline $5-6$ & 2 & 2 & $\vec{I}$ & 2 & 1 & 2 & 10 \\
\hline 6-7 & 2 & 3 & 2 & 2 & 0 & 0 & 9 \\
\hline 7.9 & 2 & 3 & 1 & 3 & 0 & 0 & 9 \\
\hline \multirow{2}{*}{$\begin{array}{l}\text { Totales: } \\
\text { Totales: }\end{array}$} & 13. & 16 & 11 & 14 & 8 & 8 & \\
\hline & \multicolumn{2}{|c|}{29} & \multicolumn{2}{|c|}{25} & \multicolumn{2}{|c|}{16} & 70 \\
\hline
\end{tabular}

dres que habían abandonado el hogar, etc. Estos niños se caracterizaban por no disponer de la figura paterna dentro de su núcleo familiar.

La muestra fue seleccionada de tres guarderías diferentes: una en el centro de la cludad, otra en la periteria y, la tercera, de una guardería social (tabla I).

\section{a) Identidad:}

- Discriminación de figuras humanas desnudas diferenciadas exclusivamente por características anatómicas unidas al sexo: 10 figuras ( 4 adultos y 6 niños).

- Autoclasiticación verbal a través de la pregunta: y tú ¿qué eres, un niño o una 
niña? (en último lugar el sexo distinto al sujeto).

- Autoclasificación por pares de figuras: presentándole por pares las figuras desnudas se le va pidiendo que conteste a la pregunta: como ¿quién eres tú, o como, quién vas a ser tú?

En los tres casos se apuntan los aciertos, entendiendo por tales la discriminación correcta o autoclasificación correcta. Las puntuaciones máximas pueden ser, por consiguiente: 10,1 y 5 , respectivamente.

\section{b) Rol sexual:}

- Discriminación de figuras vestidas y ataviadas (respecto al pelo, cara, ...) conforme a las características del rol sexual: 10 figuras ( 4 de adultos y 6 de niños de diferentes edades).

- Autoclasificación verbal por pares de figuras: presentándole por pares las figuras vestidas se le pedía que contestara a la pregunta: como ¿quién eres tú, o como, quién vas a ser tú?

- Atribución de actividades propias del rol sexual a las figuras adultas presentadas vestidas:

- hacer la comida;

- conducir;

- cuidar a los niños, y

- irse a trabajar.

La forma concreta de realización era con la pregunta «ahora todos éstos van a coger el coche y se van a ir a comer a casa, ¿quién va a ir conduciendo?», etc.

- Preferencia de juguetes tipificados:

- pistola;

- muñeco;

- cocina, y

- coche.

La forma concreta de realización de esta prueba era pedirle que eligiera dos juguetes y jugara con ellos.

En los cuatro casos se puntuó la tipificación correcta desde el punto de vista de rol sexual tal y como se expresa en nuestra sociedad. Las puntuaciones, por tanto, podían alcanzar el máximo de 10, 5, 2 y 2 .

\section{c) Conservaciones:}

- Conservación de identidad sexual de los muñecos. En este caso la prueba se escenificaba cambiando de ropa los muñecos en presencia del niño.
- Conservación verbalizada y ayudas con fotografías. Fórmula:

- ¿Qué eres, niño o niña?

- Cuando seas mayor qué serás, ¿chico o chica?

- Y cuando seas aún más mayor qué serás, ¿papá o mamá?

- Y si te vistes de (lo contrario a su identidad)... entonces, ¿qué serás?

- Si tú quieres cuando seas mayor ¿puedes ser papá (lo contrario a su identidad sexual)?

Independientemente de la aplicación estandarizada de cuanto acabamos de decir, continuábamos nuestra investigación con cada niño haciéndole numerosas preguntas y presentándole las tareas a realizar de distinta manera siguiendo un método muy similar al denominado método clínico de Piaget (1923).

Además de estos datos hicimos una pequeña entrevista a los padres y les pasamos a todos los niños la Escala de Desarrollo Brunet-Lezine (1971) y un puzzle de dos figuras desnudas con diferentes combinaciones.

Los estadísticos empleados en el análisis han sido: frecuencias, $\%, \chi^{2}$, significación de diferencias de medias dependiendo del tipo de datos.

\section{Resultados}

Los resultados, dado el diseño empleado, son amplios y complejos. Presentamos solamente los más sobresalientes:

\subsection{Factores que determinan el desarrollo de la identidad de género $y$ rol sexual:}

a) La edad:

En relación con la edad se han visto confirmados los resultados obtenidos por otras investigaciones:

- La adquisición de aspectos fundamentales del rol sexual y de la identidad sexual tiene lugar antes de los tres años:

- Sólo tres niños de los menores de dos años hizo alguna diferenciación.

- Casi todos (7 de los 10) los de edades entre 2 y 3 años establecieron alguna diferenciación de roles e identidad. Los tres 
que no lo hicieron tenian un nivel de desarrollo general, $y$, en particular, a nivel verbal, muy bajo; pertenecian a familias deficitarias y uno de ellos era gemelo (su hermano - no incluido en la muestraconfirmaba estos datos).

- La conservación de la identidad sexual la adquieren la mayoría (el 80 por 100) entre el quinto y el sexto año, en algún grado; pero aún con muy poca resistencia a la sugestión contraria. Entre los seis y ocho años la adquieren con clara resistencia a la sugestión.

La conservación autoatribuida (creer que ellos van a ser de mayores del mismo sexo que son en la actualidad) es anterior en la adquisición y más resistente a la sugestión que la conservación atribuida a otros (los muñecos, fotografía). Tal vez la razón sea el propio egocentrismo infantil. Un niño de 5 años nos lo expresó claramente: «podría ser mamá, dijo, de mayor, pero no quiero»; "no quiero ser mamá de mayor», dijo otro.

- Las preferencias cambian con la edad. Hay una tendencia cada vez más clara a elegir las personas de su propio sexo para jugar, ser amigos y quererlas más. Probablemente se trata de una roma de conciencia de la identidad y del rol sexual que en una sociedad como la nuestra, donde lo sexual va acompañado de misterio..., les haga sentirse más seguros con los del propio sexo.

\section{b) El sexo:}

En relación con el sexo de los sujetos sólo hemos encontrado diferencias en el siguiente sentido:

- La preferencia por los juguetes tipificados es más clara y consistente en los niños. Las niñas eligen más frecuentemente juguetes «de niño». La significación estadística es del 1 por 100. En los primeros cuatro años la situación es tal que las niñas como conjunto no tipifican los juguetes. Sólo a partir de esa edad lo hacen y con numerosas excepciones. Excepciones que en el caso de la muestra de hijos de «ambos profesionales» son tan numerosas que hasta los seis años no puede hablarse de tipificación de juguetes.

Por otra parte, la tipificación es más clara cuandn es atrihuida a orros y menos cuando ellos actúan sobre los juguetes; esto también en el caso de las chicas.
- Las niñas manifiestan preferencia por los miembros del otro sexo con más frecuencia que los niños: significación al nivel del 5 por 100 .

Todos estos datos pueden significar que las niñas ya perciben en algún grado el rol de privilegio de los varones, viven tal vez alguna forma de envidia o, simplemente, son más abiertas al otro sexo que los niños.

c) El nivel de desarrollo (Cociente de Desarrollo de la Escala de Brunet-Lezine) tiene una relación muy clara con la diferenciación de figuras sexuales y con la conservación. El nivel de significación es del 1 por 100. Este hecho parece confirmar la relación entre conocimiento y adquisición de la identidad y el rol, por una parte, y la permanencia de la identidad sexual, por otra, como ya postularon Kohlberg (1966) y Money (Money y cols., 1972).

No se da este tipo de relación con la preferencia de juguetes y la discriminación de tareas tipificadas, sin duda porque en este aspecto influyen más las circunstancias ambientales. Los hijos de padres "ambos profesionales» que son los que mejor nivel de desarrollo tienen son los que menos tipifican estas tareas. Esto demuestra a su vez que en «lo más cultural» es el aprendizaje social lo definitivo (Bandura y Walter, 1968).

\section{d) La familia de procedencia es un fac-} tor claramente relacionado tanto con la adquisición de la identidad como del rol sexual. Pero la relación encontrada no es la misma en ambos casos '.

- La identidad (tanto en cuanto discriminación de figuras desnudas como en cuanto a autoclasificación) es adquirida antes por los niños cuyos padres son «ambos trabajadores" y los hijos de "padre trabajador-madre en casa», que por los niños de familias deficitarias. $\mathrm{La}$ significación de la diferencia de media es clara tanto cuanto comparamos los resultados de las puntuaciones obtenidas en la discriminación de figuras desnudas $(t=3,25>2,58$; significativo al nivel de 1 por 100), como cuando lo hacemos con la autoclasificación por pares $(t=4,01>2,58$; significativo al nivel de confianza del 1 por 100$)^{2}$.

La autoclasificación como niño o niña, autoclassificación verbal, no ha podido 
ser tratada estadísticamente pues los mayores lo hacen en todos los casos con absoluta claridad y los menores de dos años, en la mitad de los casos, tienen dificultades lingüísticas para contestar a la pregunta.

Estós resultados no. significan que entre la estructura familiar y la adquisición haya una relación causa-efecto directa. Es muy probable que la relación encontrada se deba más bien a un efecto indirecto: los' niños de familias desestructuradas tieñen un nivel de desarrollo menor, espećcialmente en el lenguaje (López, 1981), y éste puede determinar también una menor capacidad de discriminación: y autoclasificación. Pero no son descartables ciertos efectos más directos: la falta de la presencia del padre, las menores atenciones educativas especialmente en el campo sexual, etc. xual:

- La tipificación conforme al rol se-

En la discriminación de figuras vestidas y en la autoclasificación a partir de figuras vestidas hemos encontrado los mismos resultados que en el apartado anterior, aunque a un nivel de significación menor ( 5 por 100). Las posibles explicaciones que dábamos allí siguen siendo útiles en este caso: el nivel de desarrollo y las atenciones educativas probablemente llevarán a que unos tengan mayor poder de discriminación que los otros.

No ocurre lo mismo respecto a la atribución de actividades tipificadas y la preferencia de juguetes tipificados. En este caso el grupo de niños cuyos padres ambos trabajan se diferencian de los otros dos. El sentido de la diferencia es: menor tipificación de actividades en niños con ambos padres trabajando fuera del hogar. Entre los otros dos grupos no hay diferencias significativas.

En la atribución de actividades tipificadas, el grupo de niños con ambos padres trabajando fuera de casa en relación con los otros dos tomados en su conjunto (puesto que las diferencias son insignificantes), tipifica menos estas actividades $(t=5,16 \$ 2,58$; significativo al nivel de confianza idel 1 por 100).

Sin embargo, no se ha encontrado diferencias significativas claras en cuanto a la preferencia de juguetes. En este caso el nivel de significación no llega al 5 por 100, aunque está cercano a él.

¿Cómo explicar esta diferencia de resultados cuando en ambos casos se trata de tipificar con respecto al rol? Ia respuesta es compleja. Creemos que han podido influir dos tipos de factores: las propias características de las pruebas $y$, sobre todo, el hecho de que si bien la mujer se ha incorporado al mundo del trabajo y lleva actividades sociales cada vez más igualitarias con el varón, esto no se ha traducido en el modelo educativo que damos a los niños. Así, por ejemplo, si empieza a ser menos extraño que un padre cuide a sus hijos, es realmente difícil encontrar padres que le compren una muñeca a su hijo.

Estos datos reflejan un hecho que aparece más claro cuando los comparamos con los obtenidos con el factor sexo: los chicos están significativamente más tipificados conforme a su rol que las chicas. Estas eligen en mayor número de casos juguetes de chico.

Lo cual, en el fondo, refleja muy claramente lo que sucede en la sociedad: los hombres están más tipificados en sus actividades que las mujeres. Cuando éstas hacen las de aquellos, eso no conlleva que los hombres hagan las de las mujeres. Así, lo captan perfectamente los niños y las niñas: mientras es frecuente que ambos (especialmente en el primero de los grupos: ambos padres trabajan) puedan trabajar y conducir, es muy excepcional que el padre sea puesto a cocinar.

\subsection{Estadios y procesos:}

- Los niños distinguen antes los roles en los adultos (fuera de ellos mismos) que en ellos mismos.

Lo primero en aparecer es la discriminación entre algunas funciones típicas de cada rol (conducir y hacer la comida, sobre todo). Sólo posteriormente lo transfieren a su actividad y a las verbalizaciones. También tipifican antes para otros que para sí mismos.

Parece con ello confirmarse el proceso descrito por Thompson (1975): primero distinguen fuera de ellos y luego «se sitúan» dentro de una categoría sexual. 
Ninguno de los niños estudiados hace el proceso inverso.

\section{- La diferenciación de figuras sexuales si-} gue el siguiente orden:

a) Primero establecen las diferencias basándose en características externas: ropa, peinado... Y por funciones: hacer la comida, conducir...

b) Segundo, establecen las diferencias por los órganos genitales. Ninguno de los niños estudiados hace el proceso inverso. datos:

Este proceso lo prueban los siguientes

- Las láminas que presentan figuras vestidas, así como los muñecos vestidos, es lo primeramente diferenciado por sexos.

La lámina de la familia desnuda, donde los padres se diferencian también por el peinado y los hijos sólo por los genitales, refleja también las dos fases: primero se diferencian los padres y posteriormente los hijos.

- En la lámina de los cuatro niños desnudos, donde dos se diferencian tambièn por el cabelio y dús súló pố lós genitales, los primeros son discriminados antes que los segundos.

- En la lámina de las partes del cuerpo, donde la cabeza está tipificada por el cabello y la barba, es la cabeza lo primeramente tipificado.

Respecto a las otras tres partes del cuerpo (pecho, genitales y piernas), las diferencias no son significativas.

- En el puzzle de contradicciones donde se les presenta una cabeza de mujer con cuerpo de hombre y una cabeza de hombre con cuerpo de mujer..., todos, hasta los seis años, hacen una discriminación por la cabeza. Sólo posteriormente afirman: "esto está mal», "no puede ser".

Antes de este período tienden a creer lo que ven y lo interpretan a partir de la cabeza. Bien significativo es el caso de mi hija de 4 años que ante esta visión comenzó a llamar a la madre y a decirle: «mamá, mira, una mamá con pito». Otros muchos niños de esta edad no se duañ cueñita du la situación: y son bastantes los que después de explicárselo mantienen que el puzzle está bien.
Sólo los muy mayores (entre 7 y 9 años), además de reconocer el error, si se mantiene esa figura y se les dice "pero si fuera de verdad así, ¿qué sería?", despùés de dudar e incluso protestar por la pregunta, afirman que los genitales serian lo determinante. Aún así se los ve perplejos. pasos:

- Ia conservación sigue los siguientes

a) Primero, conservación por características externas, conductas...

b) Segundo, conservación por los genitales.

Ningún niño de los estudiados sigue el proceso inverso.

Datos que lo prueban:

- Es el camino que siguen también en la diferenciación de género. En la conservación se reproduce, a otro nivel, el mismo proceso. Se trata de un verdadero «décalage» en sentido piagetiano.

- El proceso que siguen en la lámina del puzzle lo confirma. Solo bastante tarde anteponen en el razonamiento los genitales.

Luego los niños hacen un proceso de «conservación» desde lo. más cultural (características externas) a lo más biológico (diferencias anatómicas).

¿Es este un proceso universal o es propio de nuestra cultura? No podemos responder por el momento. Puede ocurrir que los niños habituados a discriminar por circunstancias externas transfieran este hábito a la situación de examen. En una cultura distinta podría ser de otra manera. Esto nos lo ha hecho sospechar el hecho de que los hijos de padres " "profesionalizados" y de aquellos que dicen hacer educación sexual directa con sus hijos, son los que además de adquirir roles más flexibles, también usan antes el razonamiento de los genitales como prueba de discriminación.

\section{Conclusiones}

1. La edad cronológica y, más aún, la edad de desarrollo, es un factor que condiciona en grap medida la identidad sexual, el rol sexual y. la conservación de ia identidad sexuai.

2. El sexo actúa también de forma 
significativa: los niños se tipifican de forma más rígida que las niñas.

3. Las circunstancias ambientales juegan un papel decisivo en este.proceso: a) de ellas depende especialmente el tipo de rol que adquieren. Puede decirse que los niños reproducen, casi como un cliché, lo aprendido en el núcleo familiar. b) afectan a la evolución general y a través de ella a la evolución de la identidad, rol y conservación.

4. Nuestros datos no permiten decir que lo primero en adquirirse es la identidad de género, puesto que esta toma de conciencia se hace, en primer lugar, basada en características externas propias de los roles.

Lo único que podemos decir, y en esto sí estamos de acuerdo con Thompson (1975) es que primero hay una distinción "en el exterior»y luego una autoatribución.

La discusión entre Bandura y Walter, por un lado, y Kohlberg, por otro, no parece tener mucho sentido: el conocimiento permite un juicio sobre la identidad sexual, pero éste se basa, al comienzo, más en los roles que en la valoración de los genitales.

5. Los estadios descritos en cuanto a la conservación (por características más de rol y por los genitales) se confirman en nuestro medio. pero creemos que este proceso es más bien cultural y no necesario.
Por otra parte, es muy probable que la conservación exista en algún sentido desde bastante antes (esto prueba la dificultad para aceptar los cambios de identidad después de los tres años), aunque aún no se haga un razonamiento lógico que manifieste la identidad. No debe olvidarse que la estructura intelectual de los niños anterior a los seis años es de egocentrismo (no manejan varias perspectivas a la vez) y que al cambiarle u ofrecerle una característica distinta ( $«$ si a un niño le vestimos de niña", etc.), ésta puede pasar a ser la central.

Por ello nos inclinamos a pensar que aunque falte la lógica de la conservación no necesariamente falta la conservación.

Lo mismo podríamos decir si recurrimos a otro ejemplo: cuando a un niño le decimos: «si tú quieres, ¿puedes ser una mamá cuando seas mayor?», su egocentrismo (forma de conocimiento llena de adherencias subjetivas) le lleva a decir: "claro, si yo quiero, puedo".

Por todo ello, aunque nuestro trabajo confirma otros, hay que tener en cuenta cuando interpretamos estos datos cuál es su procedencia (unos niños en una edad determinada), ya que el afirmar o comprobar que no tienen aún la lógica del adulto no significa que no "conserven su identidad».

Quisiéramos volver sobre este punto en otras investigaciones.

\section{Referencias}

Bandura, A., y Walters, R. W. (1965): Aprendizaje Social y Personalidad. Alianza U. Madrid, 1974. BEE, $\mathrm{H}$.: $E l$ desarrollo del niño. Harla. México, 1969.

DE VRIES, R.: "Constancy of generic idencity in the years three to six". Monographs of the Society for Research in Child Development, 34 (Whole No. 3). 1969.

EMMERICH, W., y otros: «Evidence for a transitional phase in the development of gender constancy». Child Development, $1977,48,930-936$.

KoHLBERG, L.: «A cognitive developmental analysis of children's sex role concepts and attitudes». En E. E. Maccoby (ed.). The Development of Sex Differences. London: Tavistock. 1966.

LÓPEZ, F.: «Niños en casa cuna». Infancia y aprendizaje, 1982. 16.

LOPEZ, F.: alnterpretaciones teóricas de la evolución de la sexualidad». Sexología, n. ${ }^{\circ} 4$, INCISEX. Madrid, 1980.

MARCUS, D. E., y OVERTON, W. F.: "The development of cognitive gender constancy and sex role preferences». Child Development, 1978, 49, 434-444.

MCCONAGHY, M. J.: "Gender Permanence and the Genital Basis of Gender: Stages in the Development of Constancy». Child Development, 1979, 50, 1223-1226.

MONEY, J., y EHRHARDT, A. A.: Man and Woman, Boy and Girl, Baltimore: Johns Hopkins University Press. 1972. 
MONEY, J.; HAMPSON, J., y otros: «Imprinting and the establishment of gender role». Archives of Neurology and Psychiatry, 1957, 77:333-6.

MONEY, J.: Asignaturas sexuales, ATE. Barcelona, 1975.

Papalia: Psicologia Evolutiva, Mc Graw Hill. Madrid, 1979.

SeARS, R. B.; RAU, L., y AlPert, R.: Identification and Child Rearing. Stanford, California: Stanford University Press. 1965.

SLABY, R. G., y FREY, K. S.: «Development of gender constancy and selective attention to same-sex models». Child Development, 1975, 46, 849-855.

THOMPSON, S. K., y BENTLER, P. M.: «A developmental study of gender constancy and parent preference». Archives of sexual Behavior, 1973, 2:379-385.

Thompson, S. K.: "Gender labels and early sex-role development». Child Development, 1975, 46:339-47.

MARGARET, N. R., y otros: Age and Sex Differences in Satisfying Love Relationships across the Adult Life Span. University of Southern California. Los Angeles, Calif., 1981.

Broughton, J. M.: The Divided Self in Adolescente. Teachers College, Columbia University. Nueva York, 1981

MCGeE, J., y otros: Gender Typing and Androgyny in Later Life. New Directions for Theory and Research, University of Oregon, Eugene, Oreg., USA; Duke University Medical Center, Durham, N. C. USA, 1982.

Douvan, E.: «Sex role learning». En Coleman, The Shool years. Methuen, 1979.

KESLER, RH.: Toward a Redefinition of Sex and Gender. Montclair State College.

\section{Resumen}

Este trabajo examina el proceso de adquisición de la identidad y el rol sexual, asi como algunos factores que condicionan esta evoluciön. Se utiliza el método clinico piagetiano, con la ayuda de imágenes gráficas.

Entre las conclusiones más importantes cabe señalar: 1) La adquisición de la identidad y el rol sexual tiene lugar, en sus aspectos más destacados, entre los dos y tres años de vida, 2) La conservación (autoaplicada) de la identidad no es alcanzada basta los 5.7 años, aproximadamente, 3) Los roles sexuales reproducen de forma particularmente directa la situación del núcleo familiar.

\section{Summary}

In this study we examine the stages children undergo in order to acquire their gender identity and sex-role, as well as the factors which condition this evolution.

Our metbodology involves the presentation of graphic images (children and adults naked and clothed, parts of the body, their various functions) following the clinical method of Piaget.

The following factors stand out from the results obtained: 1) The acquisition of sexual identity and the sex-role in its most impostant aspects, takes place between two and three years of age; 2) The retention of gender identity does not become constant until between five and seven years of age; 3) The asigned sexual roles reproduce in a noticeably direct manner the characteristics of its own familyly nucleus, specially in the very young children.

\section{Résumé}

On examine dans ce travail le processus d'acquisition de l'identité et le rôle sexuel, tout comme quelques des facteurs que conditionnent cette évolution. On utilise la méthode clinique piagetienne avec l'aide dimages graphiques.

Parmi les conclusions les plus importantes, on remarque: 1) L'acquisition de l'identité et le rôle sexuel ont lieu, sous ses aspects les plus remarquables de 2 a 3 ans, 2) La conservation (auto-appliquée) de l'identité n'est pas atteinte par l'enfant qu'a l'âge de 5-7 ans, 3) Les roles sexuels reproduisent de manière directe la situation du noyau familial. 